(25)

60 or ens

6.7. 9.920.

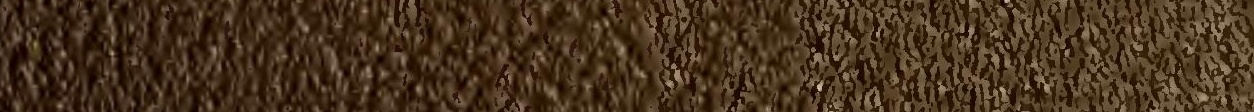

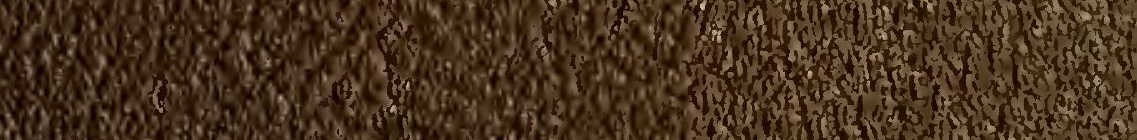

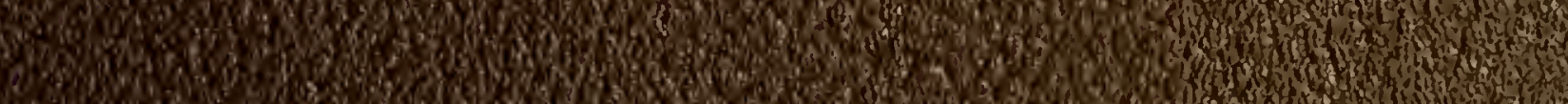

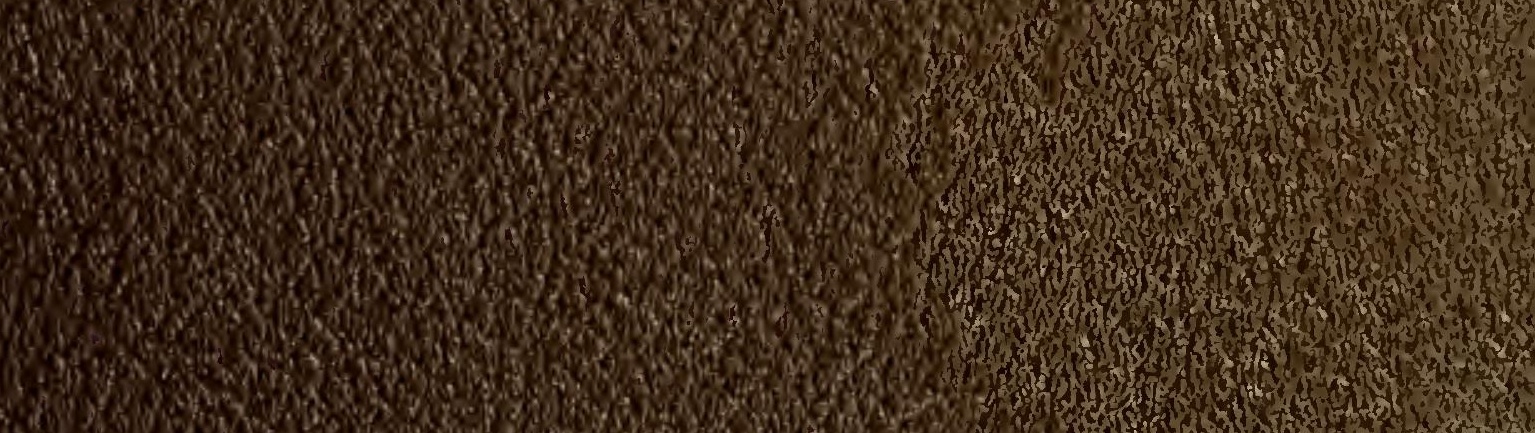
nawa W.1.

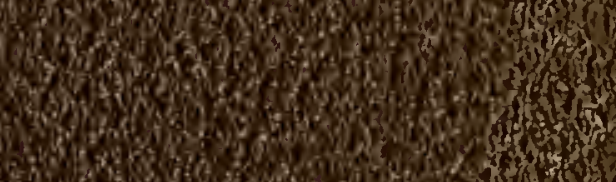
W. ons 0

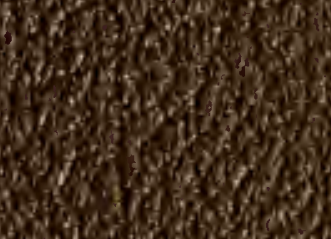

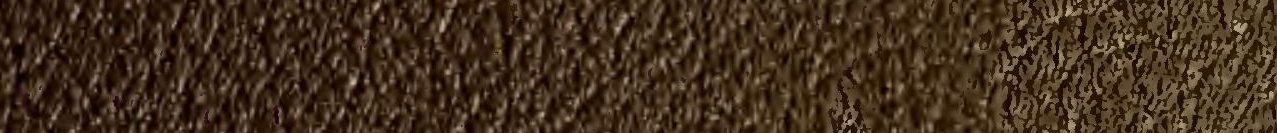

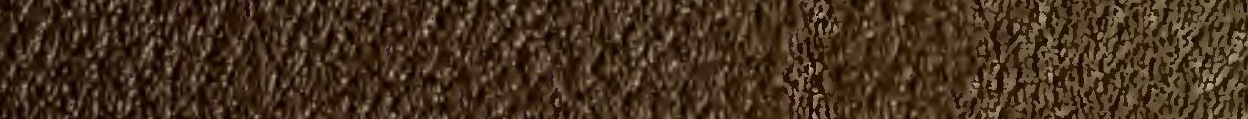

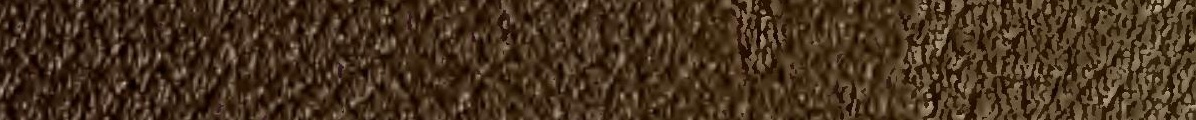

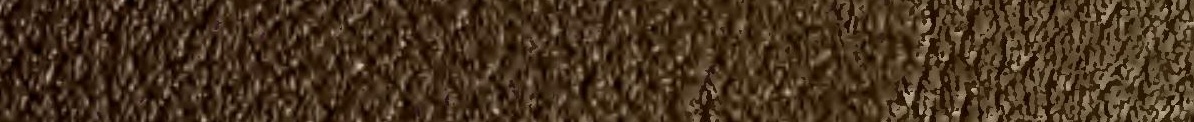

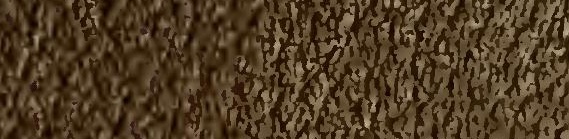


IINIIISNI NVINOSHLIIVS

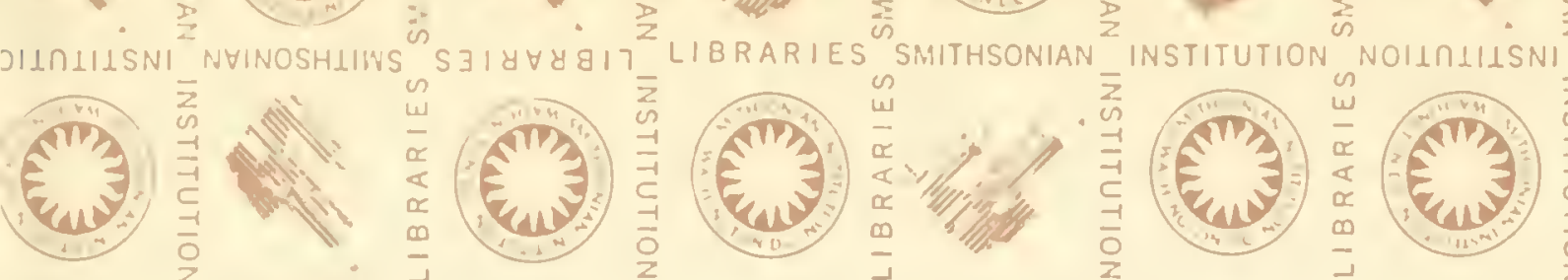

(a)

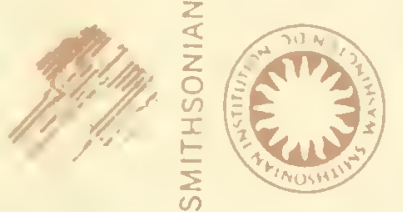

BRARIE

(in)
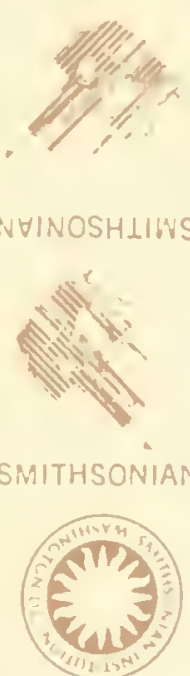

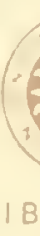

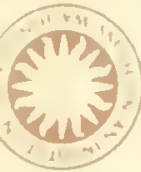

Eiin)
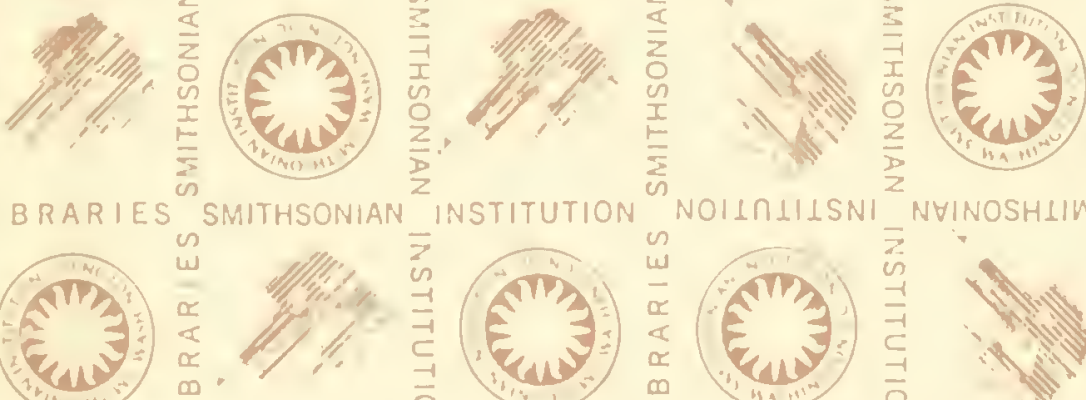
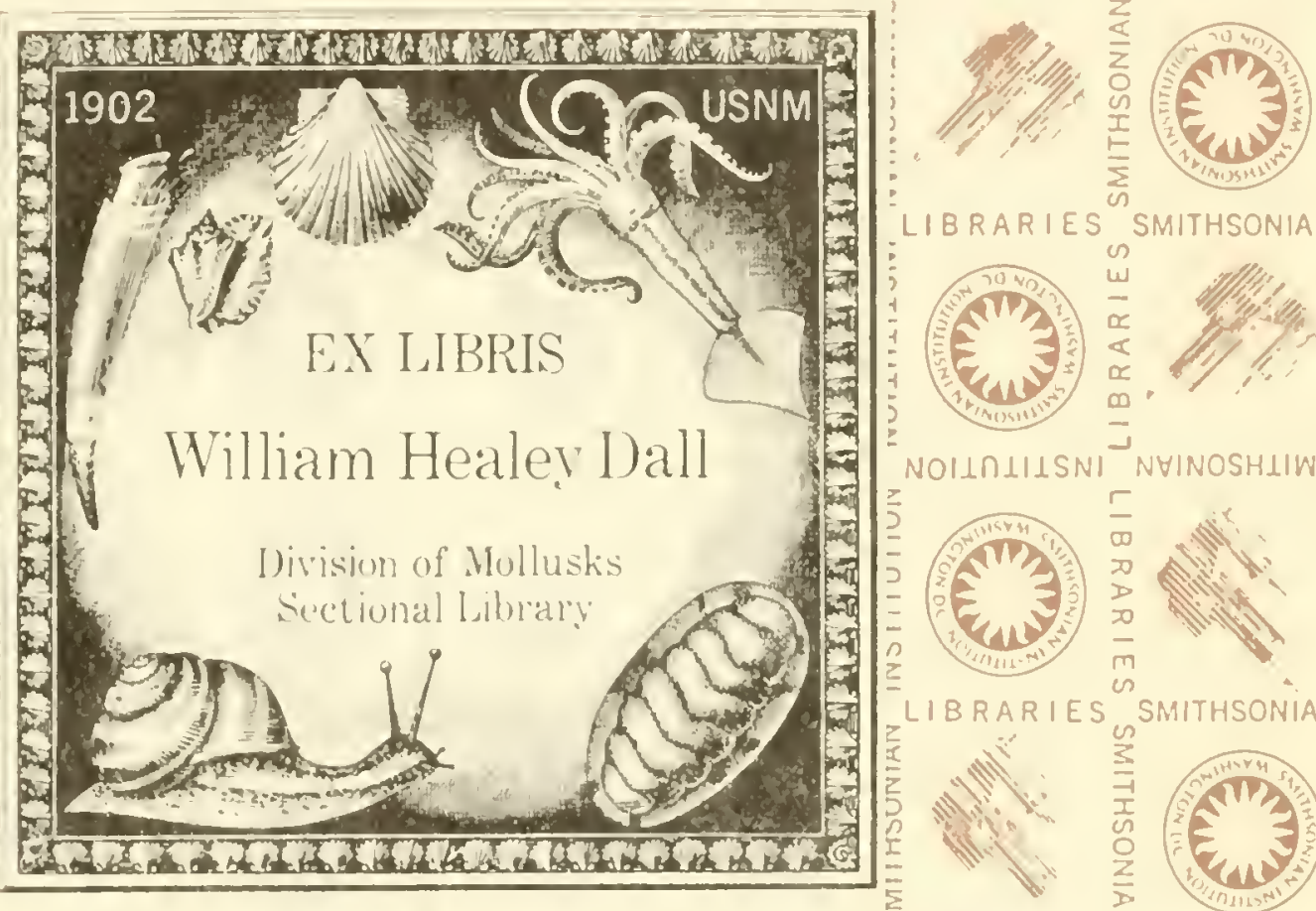

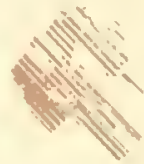

SMITHSONIAN 

Division of Mollusta

Sectional Library 



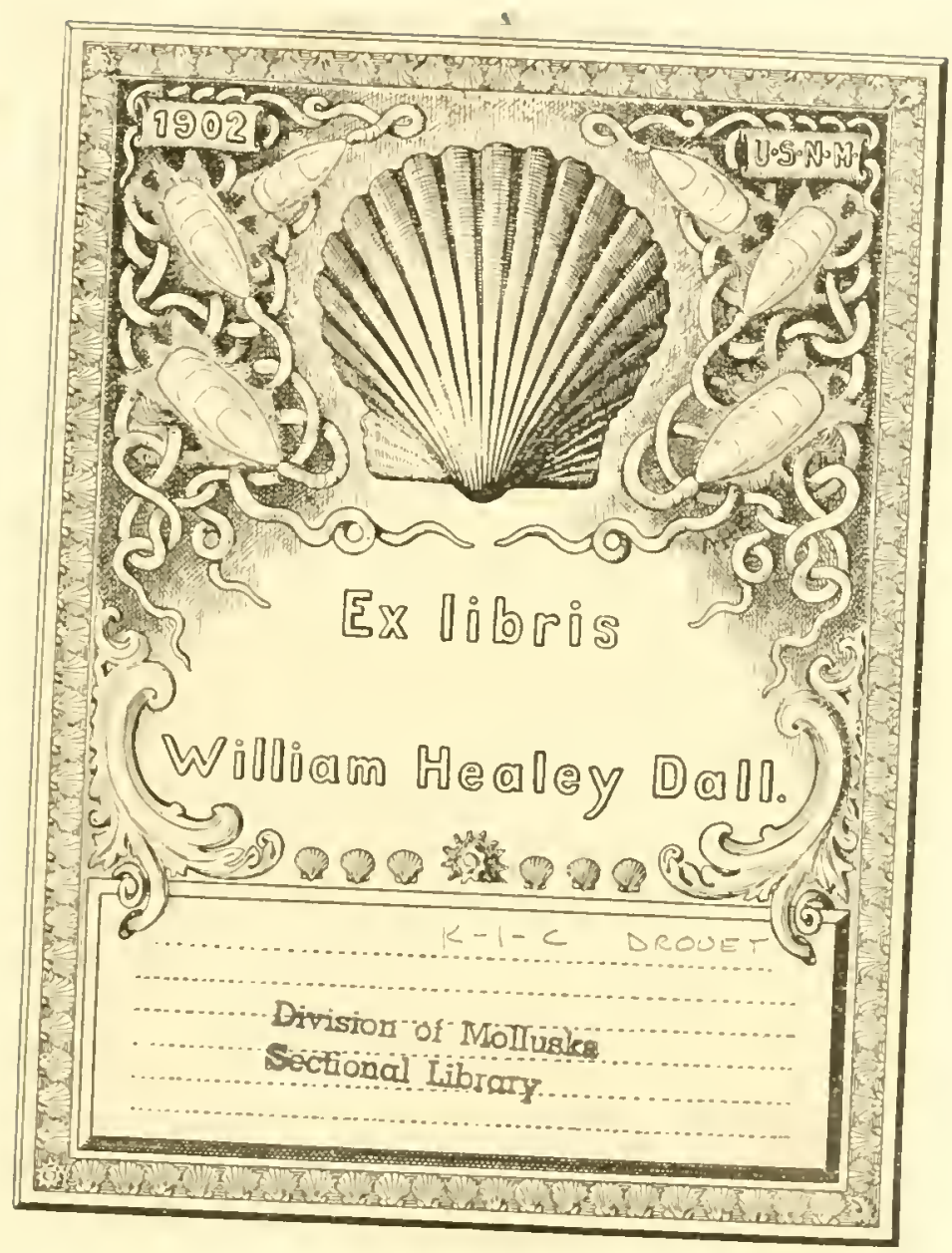





\section{MOLLUSQUES MARINS}

DES AÇORES. 
Ouvmgen de M. Dmoxír.

litudes sur les Naïmes de la Funce. Paris, 1838, et Troyes, 1857; deun parties in-80, 18 planches.

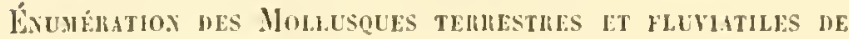

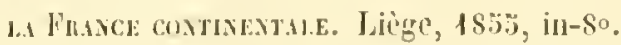

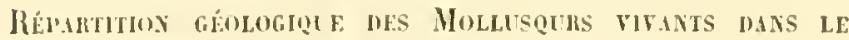

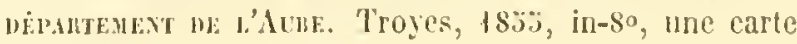
géol. color.

Letthes conchrlohoglques, Paris, 1856 , in-8o.

Rapolit a Sa Majesté le Roi me Pontugal suk un votage D'explontiox scaratifleue aUx iles Arones. Troyes, 1858 , ill- 40 .

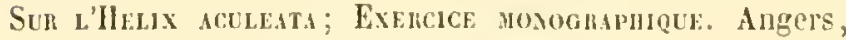
1S38, in-So, une planche coloriće.

A Troyes, chez l'Auteur, rue du Palais-do-Justice, 26; et à Paris, chez Baillizae, ruo llautefeuille, 19. 


\section{MOLLUSQUES MARINS}

DES

\section{ILES AÇORES,}

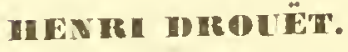

2 planches dessinées par le Dr Baudon.
Dimieios of Molinsks EAtirne' 'l, rewy

\section{PARIS}

CIEZ BAULIERE, LHRIIRE,

ruc Hantefcuille, 19.

$18: 38$. 


\section{SOIII I IE.}

Préambule.

Auteurs cités.

Mollusques marins des Açores.

\section{TABLEAU DES GENRES :}

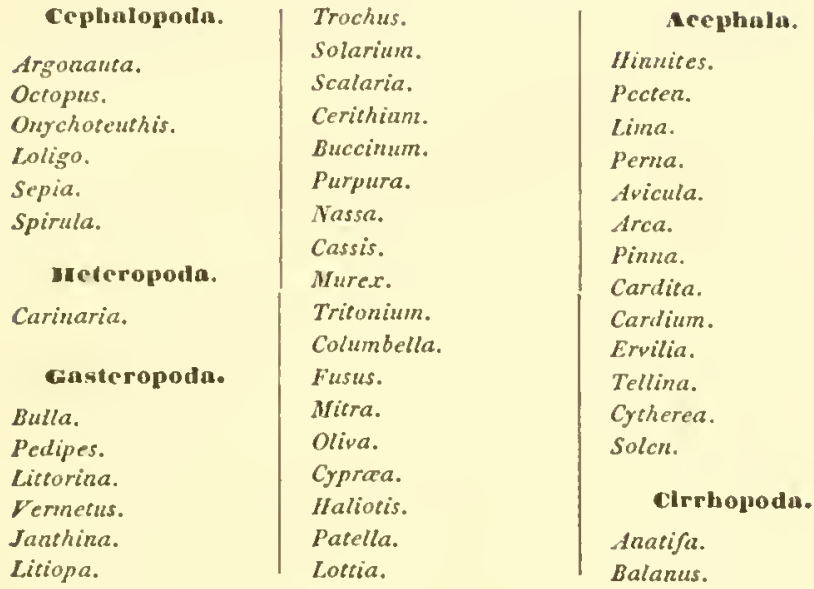

Appendice : Mollusques fossiles.

Explication des planches.

Planches. Ces deux planches, dessinées par le Dr Baudon, de Mouyde-l'Oise, représentent cinq especes nouvelles, savoir : Litiopa Gratelupeanu, - Nassa Deshayesii, - Patella Gomesii, Patella Baudonii, - Patella Moreleti, et une variété intéressante du Columbella rustica, var. Azorica. 



\title{
MOLLUSQUES MARINS
}

\author{
DES \\ ILES ACORES, \\ PA \\ AENRI DROUET,
}

Membre residant.

PRÉAMBULE.

Des quatre groupes d'îles qui se trouvent dans l'Océan Atlantique au nord de l'équateur, à une distance plus ou moins grande des côtes d'Afrique et d'Europe, les Açores et les îles du Cap-Vert paraissent avoir été jusqu'ici négligées par les malacologistes.

Bowdich, Lowe et Albers ont, à différentes époques, exploré les îles de Madère et de Porto-Santo, et ils ont donné dans diverses publications le résultat de leurs recherches en ce qui concerne les mollusques 
terrestres. On connait peu les espèces marines de ces parages.

En second lieu, Webbet Berthelot ont étudié les mollusques des Canaries; et $\mathbf{M}$. d'Orbignyy, réunissant les matériaux rapportés par ces deux naluratlistes et ceux qu'il avait lui-mème amassés, a publié un fort beau travail d'ensenıble sur la conclyyliologie marine et terrestre de cet archipel. Depuis, Blauner parail avoir exploré de nouveau ce groupe, et le résultat de ses recherelies a été mis au jour par M. Shutleworth.

Seules, les Agores et les iles du Cap-Vert n'ont encore été l'objet d'aucune publication spéciale; et pourtant ce sont peut-être les deux groupes les plus importants de cette partie du globe. Les Acores, qui apparliennent au Portugal, se composeut de neuf îles ainsi nommées: San-Miguel, Santa-Maria, Terecira, San-Jorge, Pico, Fayal, Graciosa, Florès, Corvo; elles sont comprises entre $39^{\circ} 45^{\prime}$ et $36^{\circ} 50^{\prime}$ de latitude Nord, et entre $27^{\circ}$ et $33^{\circ} 40^{\prime}$ de longitude Ouest.

Pendant le printemps et l'élé de l'année qui vient de s'écouler, j'ai exploré ces iles avec M. Arthur Morelet, de Dijon, el durant les cinq mois de mon séjour dans cet archipel où bien peu de naturalistes ont encore pénélré, j'ai recueilli les mollusques terrestres, les mollusques marins, les vertélbés, les insectes coléoptères, les zoophytes, les plantes el les roches. Mon savant compagnon de royage devant publier bientôt une histoire des mollusques terrestres que nous avons olservés (il n'y a pas une seule coquille fluviatile aux $A$ çores), je laisserai en- 
tièrement de côté cette partie de mon exploraliori, pour ne mocenper ici que des mollusques marius.

Les conchyliologues remarqueront saus doute une certaine analogie entre la faune des Acores et celles de la Corse, de la Sicile, du golfe de Naples, et il leur suflira, pour apprécier ces rapports, d'ourrip les catalogues de Payraudeau, de liequien, de Plilippi, de Maravigna el de Scacehi.

11 sera facile également de se convaincre que la plupart des espèces mentionuées dans notre opuscule se retrouvent soit dans la Méditeranée, soit aux iles Canaries, soit unfin dins la mer des Antilles, fail que la position des Acores, intermédiaire entre ces différents points, evplique panlibilement.

D'un autre côté, on sera surpris peut-ètre de irouver eelte faune moins rielre que etles des régions que je viens de citer, moins riche aussi que celles des côtes du midi de la France, de la Sardaigne, du golfe Adriatique, et de plusieurs autres contrées analogues par leur coustitution géologique el leur latitude. Spécialement, elle est plus restreiute que celle des îles Canaries, où l'on trouve, selon M. d'Orbigny, 196 espieces de mollusques, dont 1.39 marines et 57 terrestres ou fluviatiles, tandis que notre catalogue ne comprent que 75 espèces marines, dont cinq sculement sont nourelles (1); mais l'archipel canarien, par sa prosimité du tropique el du coutiuent

(1) Lourrage de M. Morelet comprendra l"indication de 70 especes emviron de mollusyues terrestres, dont près de 50 sont incidites! 
africain, se troure évidemment dans des condlitions plus heureuses.

En outre, je n’ai ohservé aucune des espèces rares du Portugal, telles que le Fusus contrarins, le Priamus stercus-pulicum, le Donax fragilis, le I'anopoa Aldrovandi, et plusienrs autres qui se rencontrent quelquefois sur les côtes occidentales de la Péninsule, et que j'avais l'espoir de retrouver dans l'archipel acoréen; enfin, il n'y a point d'lunitre dans ces parages, tandis yu'on en trouve an moins deux on trois sur les côles de Portugal, ct deux aussi aux Camaries : les Ostrea cochlear et crista-galli.

A quoi donc attribuer le peu de développement de la vie malacologique (s'il est permis de s'cxprimer aiusi) dans l'ocean qui baigne des rivages enveloppés par un eicl si doux?... Ëst-ce à la nature des roclies, qui sont toutes basaltiques et trachytiques, ou bien à la profondeur énorme de la mer le long des côtes, ou licu à la conformation même de ces côtes, qui sont accores, presque toujours à pic et sans plage, ou bien encore, ct surtout, à la violence contimuelle des ragues qui battent sans cesse les rivages avec une fureur extrème?... Sont-ce tontes ces causes réunies qui prodnisent ce résultat?... Jc ne saurais me prononcer; mais je ne puis mienx comparer la rareté de la vie animale sur les côtes de cet archipel qu'à celle reprochée, par M. de Quatrefages, à certains points de la Sicile (1). J'ai quelquefois parcouru plusieurs lienes de cûte et des plages d'une

(1) Sonvenirs d'un naluraliste. Paris, 18.54; 2 vol. in-18; passim. 
certaine étendue sans roir une scule coquille, soit vivante, sur les rochers, soit morte, sur le sable, apportéc par les llots.

Cependant la température de la mer est extrêmement douce et égale dans ces parages, et elle est encore entretenue dans cet état par le passage du Gulf-strean, dont la présence a élé signalée an nord et à l'ouest de l'archipel (1).

J'avais d'abord pensé an manque de régétation sous-marine comme pouvant ètre une des causes de la rareté des mollusques dans ces localités. Mais j’ai bien vite abaudonné cette idée quand j’ai songé aux masses énormes de Fueus dont on a conslaté l'existence dans ces mers (au point de les faire nom. mer mar de Sargasso!), el quand j'ai pu vérifier moimène ce fait autour des iles de Florès et de Corro, oì la mer est lifléralement couverte d'algues à certaines époujues de l'année (2).

Peut-être aurais-je fait une moisson plus abondante si j’arais pu mettre à profi la renconlre que je fis de

(I) Voyez : de Ilumbold, Voyage aux régions riquinoxiales du Noureau Continent; Tublean de la Nuture, itc. - J'il lecueilli, sur les côtrs de Florès, des graines de Jlimosa scandens charries par ce courant depuis l'amerique tropicale.

(2) Les Acoréns donnent le nom de Sargasso à plusieurs plante's marines de la famille des Algues et de l'ordre des Fucus, parliculicrement aux Surgassum vulgare. Ig., baccifurum.Ig. et stenophyllum dart. Hans les parages de Flores ot de Corvo, la mer est quilyurfois courrerte d'une telle quantité de ces Fucus, que les illsulaires l'ont appulée mer de Sargasso fmar de Sar. gasso; cest ce que les ancions navigateurs rspagnols nommaient des mairies: praderias de yerra). C'est arec ces plantes. de petits conuillages et des liagments de polypiers que les jeunes 
ces prairies de la mer, comme les appellent les anciens auteurs espaguols, et si j'avais pu porter mes investigalions à unc cerlainc profoudeur. Mallıcureusement, les circonstances ne nínt pas servi pour tout eela, el mes recherebes ne se sont guere élendues qu'aux especes qui habitent le littoral.

Je me suis adressé anx pécheurs, qui sont nombreux, notamment dans les iles de San-Mignel el de Pico; mais leur concours a élé, je puis le dire, on ne peut pas plus faible. Ils ne comprenaient pas qu'on puit venir de si loin pour ramasser de semblables choses, et je n'ai jamais pu les décider, ni pour or ni pour argent, a me rendre, sous ce rapport, de vériblbles services. Ils n'aiment pas, d'ailleurs, il rien changer à leurs habitudes. C'est un bècheur de Pico qui, me voyant déployer une certaine ardeur a la reclıerche des petits mollusques terrestres, dil un jour confidenticllement à mon muletier : "C'est bien dommage, mais ce seigneur français est pris de la! n et en disant ees mots, il se touchait le front d'une manière très-significative.

Au reste, il faut hien le reconnaître, pour étudier convenablement la conclyliologie marine, il est indispensable d'habiter longlemps une contrée. Ce n'est pas au hout de quelques mois de séjour et de recherches plus ou moins suivies, qu'on peut se flatter de connaitre la faune d'une région uu peu éten-

filles de Fayal (Iesquelles, pour le dire en passant, sont avec celles de San-Mlignel les plus industrieuses et les plus adroites de l'archipel) composent, avec infiniment de goùt et d'habileté, des groupes tres-remarquables pal l'heurense combinaison des couleurs et la délicatesse des matériaux. 
DES ILES ACORES.

due, surtout d'un archipel. L'on pourra signaler les espèces vulgaires et sédentaires, mais combien d'espéces rares ou voyageuses vous auront échappé! II est done nécessaire de ne considérer ee calalogue que comme un premier essai, un premier jalon planté sur la route, indiquant les mollusques marius les plus communs des Acores, et je ne serais point élonné si le nombre en étail un jour doublé. D’ailleurs, j'aurais pu enrichir ma liste de plusieurs autres espèces signalées dans des régions voisines et analogues; mais je ne l'ai pas voulu fare, me boruant a indiquer ce que j’ai moi-même observé.

Quoiqu'il en soit, il est à remarquer que cerlaines espéces prédominent dans ces parages, et reparaissent fréquemment sur les côles au préjudice ou à l'exclusion de toutes autres. Elles y sonl presque toujours réunies par groupes assez nombreux, el depuis longtemps déjà les indigènes ont tiré parti de ces espèces sédentaires, dont la prise de possession des localités est parfaitement assise et assurće, au point de vue de l'alimentation. Telles sont, par exemple, le Patella crenata (et les autres espèces du genre, qui peuvent être regardées comme les mollusques essentiellement prédominants sur les cơtes açoréennes!), le Balanus cintinnabulum, le Purpura homastoma, le Littorina striata, toutes édules, et très-abondantes sur presque toutes les côtes de l'archipel; telles sont encore l'Arialifa lavis el les Maliolis, non moins répandues que les précédentes; et enfin, les Pecten pusio, Cardium fasciatum et Columbella rustica, qui pullulent également sur le littoral de plusieurs îles.

D'autres, au contraire, sont fort rares et ne se rencontrent pour ainsi dire qu'accidentellement, 
amenéce de lointains rivages par les conrants, ou soulevées des profondeurs de l'Océau par la lempùte. Tel est le cas, par exemple, du Carinaria fragilis, dont on ne connail guere qu'une capture ou denx près de l'archipel, du Jemthina exigna, du Solerimm lutem, du Nassa Deshayesii, des Litiopa mitidula et Gratelupeana, du Solen marginatus, et de quelques autres encore, qui ne sont que de passage ou aecidentelles, si l'on peut s'exprimer ainsi.

Cependant, de la rareté des mollusques en général, il ne faudrait pas conclure a la compléte extinetion rle la rie animale dans la mer des Açores et sur ces côles éminemnent volcaniques, trop souvent ¿́prourées par des tremblements de terre.

C'est ainsi que l'on rencontre fréquemment dans ces parages plusieurs grands mammiféres de l'ordre des Célacés, notamment le Catodon macrocephalus (on eachalol), qui fail l'ohjet d'une pêche active el étendue de la part des baleiniers américains, le Phocuna commumis (marsouin) qui est abondant, et d'autres espèces des genres 1)elphimus, Mhyseter el Balonoptera. Une cenlaine environ de baliments croisent annuellement dans la mer des $\Lambda$ cores pendant la belle saison, c'est-à-dire depuis le mois d'arril juşu'en novembre, et se livent exclusivement à celle péche, ou phulot a ce genre de chasse, qui a ses daugers.

L'on péche souvent, et l'on mange une fortue d'assez lorte taille el de grands crustacés correspondarst al nos liomards et à nos langoustes.

L.es poissons sont nombreux en espèces el extrêmement abondants en individus. C'est la base de la nourriture de tomles les classes, riches el paurres, et la profession de pecheur est, entre toules, celle qui 
DES ILES ACORES.

compte le plus de représentants. Chaque matin, des centaines de barques s'éloignent de la cóle et ront demander aux innombrables plaalanges qui sillonnent l'Oeéan un pain quotidien. Quelquefois la pêche a lieu pendant la nuit, surtout pour les tortues, les langoustes el les lımards, et plus d'une fois j'ai vu les côles éclairées, de distance en distance, par des feux dont la signification m'était d'abord inconnue, mais que le navigateur voit toujours avec plaisir. "II n'est pas un voyageur, dit M. Berthelot, qui, en traversant l'Atlantique, n'ait observé comme moi ces brillantes coryphènes et les honites légères qui se jouent dans le remou, les poissons pilotes qui s'altachent au raisseau et se plaisent dans son écume, les légions de llons, dont la pèche providentielle fait la joie de l'équipage, et ces dauphins navigateurs que le marin signale de loin comme un heureux présage : arant-coureurs d'un vent frais, ils arrivent du bout de l'horizon, hondissent sur la lame comme pour saluer le navire, plongent sous sa quille, le croisent dans sa marehe, s'éloignent et resiemment dans un clin-d'cuil pour recommencer vingt fois leurs évolutions. Et le terrible requin, aux sinistres traditions, toujours de l'arrière, prêt à engloutir ce que la fatalité, le hasard ou la ruse viendront offrir à sa voracité (1). " - Ces poissons paraissent appartenir plus particulièrement aux genres Zeus, MU gil, Thymnus, Zigana, Xiphias, Serramus, Scomber, Caranx, Sparus, Boops, elc. Je me souviens d'en

(1) De la Péche sur la cote occidentale d'A frique, par Sabin Berthelot. Paris, 18:40; in- $8^{\circ}$, page 57. 
avoir vu sur lea marchés qui ressemblaient, pour la forme, a de grands serpents marbrés tle noir et de jaune.

l.es polypes el les coraux paraissent aussi assez réprandus, surtout autour de San-Mligucl, de Fayal et de Pico. Rien de plus élégant que le Gorgonia verrucosa et plusieurs autres especes coralligènes qui étendent leurs rameanx tantôt flexibles, tantôt rigrides, comme des palmes délicates ou comme des fleurs pétrifiées.

On rencontre encore assez communément plusieurs échinides ed stelléricles, molamment les Eichimus brevispinosus et lividus, dont les baigneurs redoutent les pointes acérées, ef les Asterias glacialis et lerrigata (rulgairement étoiles de mer), dont les formes insolites causent un certain effroi à l'ignorance et à la crédulité.

Fufin la mer est sowrent parseméc, sur des espaces considérables, d'acaléphes de loutes les couleurs, surtout de Méduses jaunàtres el de biéroés.

La géograplic zoologique est une science d'une hate importance et d'un intérét majeur : elle indiepue la sphèe d’activité de chaque espèce animale, el circonscril les limites qui lui sont propres. C'est une des branclies principales de la phiblosophie de l'histoire naturelle, et son perfectionnement se poursuit, de nos jours, avee une louble ardeur. Tel est le lut que se sont proposé tons les auleurs de catalogues locaux (Iravail ingrat cl aride!); tel est celui que je me propose encore aujourd'hui en publiant 
DES ILES ACORES.

le Catalogue des Mollusques marius des iles A çores, pour lequel j’ai adoptcila classification jorposće dans le Genera de Sander Rang.

Je dois une mention spéciale à M. Enxesr no Casto, de San-Mlignel, pour l'obligeance avec latquelle il m'a secondé dans mes recherehes el fait part de ses propres décourertes: je la lui doune avec le sentiment d'une amirié sincère el le plaisir de la reconnaissance.

Le savant M. Desmures, de Paris, a bien roulu se charger du soin de réviser et de nommer la plupart de mes espèces : je saisis arec empressement cette occasion de Jui témoigner publiquement ma gratitude, et je suis heureux de pouroir m'appayer sur l'autorité de son nom el de son lalent, comme la garautic la plas sure de l'exactilude des déterminations spéciffques inscrites daus eet opuscule.

Arcis-sur-Aube, noùt 1858. 



\section{PRINCIPAUX OUVRAGES CONSULTES}

et

\section{AUTEURS CITÉS.}

Anusos. Histoire neturelle du Sénégal. Coquillagrs. Paris, 17.37; in-io aver planches.

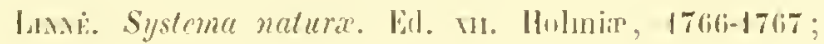
3 vol. in-so.

Guelix. Systema naturex. EH. xHI. Leipsig, 17SS-1790; 10 rol. in-So.

Bnuguibr. Histoire naturelle des Vers. Encyclopidic methodique, tone VI. l'aris, 1789-1792; in-fo av. pl.

Moxtage. Testacea Lrilannica. London, 1803 ; 2 vol. ingo ar.pll.

linocc.⿲I. Conehyliologia fossile subapennina. Milan, 1 S14; 2 vol. in-in.

Lannek. Wistoire naturelle des Animaux sans vertébres. Paris, 1SI5-1822; 7 vol. in-so.

Finuserac. Tablecux systimatiques des Animanx mollusques. Paris (1821); in- 90 .

Turtox. Conchylin insularum Britannicarum. Exeter, 182.; in- ;o alv. 11.

Puratenese. Catalogue deseriptif el methodique des Annélides et des Hollusques de lile de Ciorse. Paris, 1826; in-so all. pll. 
Risso. Histoire nalurelle des principales productions de I'Europe méridionale. J'aris, 1820; ; isl. in-So av. pl.

Risa. Manuel de l'hisloire nalurelle des Mollusques. Puris, 1829 ; in-18.

A. wOrbug. Mollusques, Eehinodermes, Foraminiferes et Polypicrs, recucillis aux ilrs fianaries par. I/ H. Wrbb

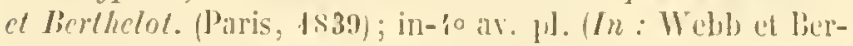
thelot, Histoire naturelle des iles Canaries).

Birere. Conchologia iconica. London, 18.33-1858; in-4o ar. pl.

Dhiner. Index molluseorum que in ilinere ad Guineam inferiorem collegil Georgius Tams. Cassel, 1533; in-?o ail. pl.

A. I'Ornigvr. Mollusques virants a fossiles. J'illis, 183̈3; in-So. (Vulume contendut lia .Monographic des Ciphalopodes accilabuliferes.) 


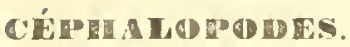

Gerre Argonate, Argonata Lam.

1. Argonautu Argo Lix.

Celte belle espèce, répandue dans les collections, et connue vulgairement sous le nom de Nautile papyracé, se rencontre de templs à autre anx Acores, surtout dans les parages de Fayal et de Pico. J'en ai v'u de très-grands excmplaires chez M. Dabncy, à Fayal, ainsi que de charmants polypiers. L'Argonaute est un des plus beaux animaux marius que l'on puisse observer. Sans parler de la coquille, qui est l'élégance même, l'animal est blanc daus toutes ses parlies, mais orné de fines mouchetures et pointillures des couleurs les plus vives et les plus agréables. Il y en a de rouges, de brunes, de violettes, lie-de-vin, argent, or, bleues, et plusicurs autres nuances; le tout disposé avec art, suivant les diverses parties du corps. L'exemplaire que j'ai rapporté a été capturé prés d'Horta, el il virail encore quand je le reçus à Pico. En détachant l'animal (que je désirais conserver dans l'alcool) de sa fragile enveloppe, je trouvai dans celle-ci une masse d'oufs, petits, ovalaires, d'un blanc un peu laiteux. Certains tests mesurent jusqu'à 2 décimètres de grand diamètre. 


\section{Genre Pourpe, Octorus Lam.}

2. Octopne vulgarls Lay.

Grande et belle especce, d'un gris violet, marbrée de taches de rouille foncées. Hlauteur : 5 décimètres, et même plus.

Habite en abondance les côtes de San-Miguel; édule pour le has peuple. Les péchenrs m'ont dit que ce mollusque répandail une lumière phosphorescente pendant la nuit, mais je n'ai pu m'assurer du fail.

Observation. J'ai tout lies de croire que ce n'est pas là la seule espèce du genre Oclopus (ou même des genres voisins) qui se trouve aux Açores. On m'en a signalé une autre plus petite et d'une couleur différente, que je n'ai pu réussir à me procurer. Je suppose que c'est l'Octopus Cuvicri d'Orb. qu'on rencontre également sur les côtes des îles Canaries.

\section{Gente Oxychoteuthis, Onychoteutmis Lich.}

3. Onychotenthle curdlopleru P'́n. (LoLigo).

M. d'Orbigny (Mollusques des iles Canaries, 1839, p. 25) désigne ce Céphralopode comme se trouvant dans les parages des Açores, principalement dans les bancs de sargasso; je l'indique sur la foj de eet auleur. 


\section{Gente Calman, Loligo Lam.}

4. Hollgo Vulgarl's LAM.

Espèce très-connue, commune dans l'Océan Allantique. Les pêcheurs de San-Mliguel la caplurent fréquemment : je l'ai vue sur le marché à Ponta-Delgada, avee l'Octopus vulgaris.

$$
\begin{aligned}
& \text { Genre Seicine, Sepia Lin. } \\
& \text { 6. Sepla offelualls Lix. }
\end{aligned}
$$

Avec la précédente : côtes de San-Miguel et de Pico.

\section{Gente Spirde, Spindza Lam. \\ 6. Gplrula peronll LaM.}

Quelques rares exemplaires ont été recueillis dans la petite baie de Rosto-de-Cào, à San-Miguel, par M. Ernesto do Canto.

La présence de cette espéce, sous celte latitude, est un fait intéressant. Lamarek ne l'avait signaléc que comme appartenant aux mers australes et à l'Océan des Moluques. Depuis, d'Orbigny l'a rencontréc en grande abondance aux Canaries, à SantaCruz-de-Ténériffe (Mollusqucs des Canaries, 1839, p. 24 et 25), et elle est indiquéc par Dunker (Index moll. itin. ad Guineam, 1853, p. 1), comme ayant élé recueillie dans le voisinage de l'île Saint-Vincent. 
Suivant d'Orligny (Moll. viv. al foss. 1855, p. 316), cette coquille serait mème jetée quelquefois sur les cótes d'Europe par les veuts et les courants. C'est ainsi que, de jour en jour, l'aréa des espèces tend à s'agrandir et prend me extension nonvelle.

\title{
MÉTÉRUTOUES.
}

\author{
Geure Campane, Campana Lam. \\ 7. Carinaria frugilis Las.
}

J'ai vu, chez M. Dabney, un exemplaire de cette rare el curicuse espéce. Il avait été capturé dans la mer des Açores, non loin de l'ile de Fayal. C'est a peu près, m’a-1.on dit, la seule rencontre qui en ait été faite dans ces parages. Quroique je n’aie pas rap. porté cette coruille, et que ce soil de mémoire et d'après mes notes que je la désigne ainsi, je crois pouvoir affirmer que ma détermination est exacte.

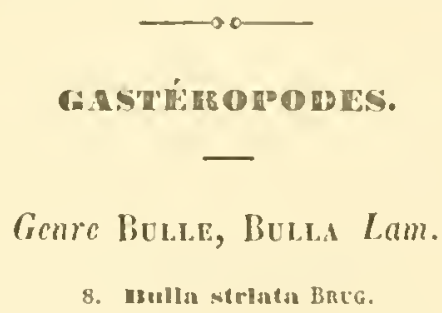

Hahite les rivages de rayal et de Pieo. - Me paraît une variété cliez laquelle les stries earactéristiques du type sont à peine apparentes. 


\section{Genre Piétix, Penpes Alans.}

9. Tecllpes ntrn Fér.

Jolie pelite coquille de forme ovoüde, imperforée, profondément et régulièrement striée dans le sens de la spire (stries espacées), d'un gris jaunàtre, terne, solide; six tours de spire séparés par nne suture peu sensible, le dernier lrès grand; ouverture grimaçante, munie de quatre à einq dents ou lames d'un blane de lait trés-luisantes, savoir : deux plis et une lame très saillante sur le bord columellaire, el une ou deux dents sur le bord droit, lequel est tranchant quoique légèrement épaissi. Suivant Adanson (Ilistoire naturelle du Sénégul; Coquillages; p. 11, pl. 1; 1757) qui, le premier, a fait connaitre le Piétin el qui l'a très-bien décrit el figurć, la grosse lame de la columelle partage le pied de l'animal en deux talons, séparés par un large sillon transwersal.

Ce mollusque habite le creux des rochers au bord de la mer, à Pico, dans la partic occidentale de l'ile. Il peut rester un certain temps hors de l'eau, car les rochers sur lesquels on le rencontre sont tantôt envahis par les vagues et tantôt à découvert. Il vit en compagnie de l'Auricula a qualis Lowe (1) (que, par parenthèse, je suis porté à considérer comme un mollusque tout aussi marin que le Pedipes afra), et

(1) L'Aurieula equalis, ainsi que je viens de le dire, vit avec le Pedipes afra, sur les rochers au hord de la ner, particulièrenent prés des sources d'eau sambatre. Selon la violence des ragues et les marée. ces rochers sont tantót submer- 
se nourrit des algues qui croissent en abondance dans ces localités.

\section{Gente Littonise, Littonisa Fér. \\ 10. Littorlun cirrulencena Lay. (Teniso).}

Habite sur les rocher's, le plus souvent hors de l'eau, à Santa-Maria et à Pico. Peu abondante.

Le Lillorina Basterolii, décrit par Payraudeau, (Mollusques de l'ile de Corse, 1826; p. 115, pl. 5, fig. 19-20), paraît n'ètre qu'une variété différemment colorée de cette espèce.

\section{I.lttorlua strlatn Kixg.}

IIalite les còtes de San-Miguel et de Pico, sur les rochers submergés. Très-abondante. Les Açoréens la mangent et l'appellent caramijo.

Facile à distinguer de la précédente espèce par sa taille beaucoup plus forte et par les cótes transversales dont sa surface est ornée. Le bord droit du péristôme est légèrement plissé-ondulé.

\section{Genre Veruet, Veruetos Aclans. \\ 12. Veruictua trlqueter Li.Y. (SERPtLA).}

Habite fixé sur les rochers et sur d'autres coquilles plus grosses (le Pinna rudis, par exemple), à

gés, tautơt laissés à découvert. Que penser de la place à assigner à un mollusque vivant dans de semblables conditions? Est-jl aquatique, est-il terrestre, ou n'est-il pas plutỏ amphibie?... 
DES ILES ACORES.

San-Miguel. - Deux variélés : l'une d'un jaune rosé, très-còtelée; l'autre blanchàtre, moins rugueuse (peut-être une autre espèce?...).

\section{Goure Jaximive, Jaxtuxi Lam.}

13. Janthina commonis List.

Cette élégante coquille, violette et blanche, se rencontre assez fréquemmeul sur les côtes, à Pico. Quelquefois on trouve, implantés sur la suture du denxième tour de spirc, de très-jeunes individus d'Analifa.

L'animal, qui est d'un violet foncé, répand, quand on le tourmente, une bave al,ondaute, incolore, assez consistante; et en outre, au bout de quelque temps, une belle teinture violelle.

Les gens de Pico appellent ces mollusques agoa riva (eau vive), et ils prétendent qu'ils s'attachent aux jambes des baigneurs.

\section{Eanthias exigun Las.}

Très-petile coquille ovale-conoïde, suhperforée, finement el régulièrement striéc-côtelée dans le scns longiludinal (ces stries forment angle au milieu du dernier tour de spire, ee qui fail paraitre la coquille comme très-légèrement carénéc), violetle et blanchàtre dans la partic supéricure du dernier tour, mince el fragile; cind à six lours de spire convexes, suture assez profonde, dernier tour trés-grand, les deux premiers lisses et brillants, sommet assez aigu; colunelle rectiligne, perristome tranchant, sinué. - Hauteur : 5, diamètre : 4 millimètres. 
Rare; deux ou Irois individus trourés à P'ico, sur les rochers au bord de la mer, avec le Pedipes afra. J'en ai ru encore quelques exemplaires sur les groupes en sargasso (1) que composent avec tant d'art et d'adresse les jeunes filles de Fayal. Facile à distinguer de la précédente par sa taille beaucoup plus petite el par l'absence des stries transversales.

Observation. - Les Janthines sont des mollusques floltants. Je les ai vues souvent dans le volsinage des côtes tenir leur coquille renversée comme pour ramper a la surface de la mer, et se laisser aller au gré des vagues, en altendant sans doute la rencontre de quelque plante. Souvent aussi elles deviennent la proie d'animaux supéricurs en force ou en gloutonnerie.

\section{Genre Litope, Litsops Rang. 15. H.Itopa nilitula l'FEIFF.}

Testa ovato-turrita, solidula, lovigata, nitide alba vel flavida, sxpe punctorum rubrorum seriebus ornata; anfractibus sex convexiusculis; columella torta, Iruncala; labro subincrassalo. - Long. $21 / 2$, diam. 11/4 lin. (Pfeiff. Wiegm. Arch.1840, I, page 255).

Sur le sargasso, à l'ouest et au sud de l'archipel des Açores. Rare.

16. I.luopa cratelupeana NoB. (Figurie)

Testa oblonga, acutissima, lucida, cornea, anfractibus 8-9 sutura superficiali separatis, ultimo maximo, aper-

(1) Voir le préambule, page 11, note en bas de page. 
tura obliqua, angusta, marginibus distinctis. - Haut. 5 , diam. 2 mill.

IIabite sur le sargasso, entere Florès el Corvo; peu abondante. Je dédie cetle espèce à mon excellent collégue et ami, M. le docleur de Grateloup, de Bordeaux, auteur de plusieurs travaux remarquables sur la conchyliologie.

$$
\begin{aligned}
& \text { Genre Troeue, Trocuos, Lin. } \\
& \text { 17. Trochus conulus Lix. }
\end{aligned}
$$

Habite la côte méridionale, à San-Miguel.

Coquille variable dans sa coloration; le plus souvent elle est d'un roux hrunitre avec des taches bleuâtres.

$$
\text { 18. Trochus eryfuroleucos GMeL. }
$$

Coquille petite, conique-aiguë, imperforée, fortement strjée-côtelée dans le sens de la spire, avec des pointillures entre les stries, surtout à la région supérjeure, roussâtre ou rosâtre, avec de pelites flammules jaunes ou blanches; sommel aigu, rougê̂tre; péristóme rose. - Hauteur: 10, diamètre de la base : 5 millimètres.

IIabite San-Mliguel, particulièrement sur la côte méridionale.

\section{Trochus unngua Lir.}

Quelques individus jeunes ont ćlé recueillis dans la baie de Rosto-de-Cào, à San-Miguel.

20. Trochus .....

En outre des trois espèces qui précèdent, j'ai re- 
cueilli dans le sable, à Pico, un Trochus plus grand et distinct, mais tellement roulé el endommagé, que sa détermination spécifique a élé impossible.

\section{Gente Campax, Solanicu Lam. \\ 21. Nolurintu Intcum LAy.}

Coquille sous-conö̈de, ombiliquéc (ombilic orné de crans blancs), lisse, bicarénée, jaunatre, avec des taches blanches sur les carènes, plus pâle en dessous; cinq tours despire convexes; bord columellaire blane, un peu réfléchi. - Hauteur: 5 , diamètre: 7 1/2 millimètres.

Habile la côte méridionale de San-Mliguel. Trésrare.

\section{Genie Scalairl, Scalaria Lam. \\ 22. Nenlarla pseudosenlarls Risso.}

Cluarmant coquillage à test brun orné de côles blanches, très-élégantes; sommel blanc, très-aigu; péristôme blanc, bordé. - Hauteur : 13, cliamètre grand: 6 millimètres.

Habite les cótes de San-Miguel.

\section{Genre Cénite, Crenituium Adans.}

23. cerluhiuแ zebrum Kir.s.

Un exemplaire, roulé el décoloré, a été recueilli dans le sable à Rosto-de-Cào, de San-Miguel; un autre à Magdalena, de I'ico. 
21. Cerithim tubcceulare MoNT.

Elégante et jolie petite coquille subfusiforme, grisitre et brunitre, fortement striće transversalement, et côtelće tuberculeuse; ouverture rétrécie aux deux extrémités, canaliculée inféricurement; \$-9 tours de spire, sulure très-superficielle. - IIauteur : 10, diamètre : 4 millimètres.

Habite Fayal. Rare.

\section{Genre Buccix, Buccisulı Adans. \\ 25. Hucchum rolgulum GaEt.}

Gmelin (Syst. nat., éd. xır, p. 3496, n 120) caractérise ainsi cette coquille, commune, suivant lui, anx Acores : Tesla ventricoso-oblonga, striis longitudinalibus pliciformibus transversas subtiliores undulatas decussantibus, et il cite des figures de Séloa et de Martini rapportées au Buccinum reliculatum $\mathbf{L}$.

Dilwyn (Descr. Calal., 1. II, p.637, n 120) rapporte ce Bucein au $B$. reticulatum de Linné, et il cite des figures de Pennant et de Da Costa qui cadrent également avee l'espèce linnéenne. Il indique les Açores, d'après Adanson.

Adanson (Ilist. nal. Sénég., p. 114, 1. 8, f. 9) lui donne le nom de Covet, et l'a observé à Fayal.

Je n'ai pas vu ce Bucein aux Açores.

\section{Gente Pocapre, Purpora Lam.}

26. Parpura hamastoma Lix. (Beccixer).

Très-commune sur les rochers du littoral de San- 
Miguel el de Santa-Maria. Se trowve également dans les autres iles de l'archipel. Edule. Les indigénes nomment ce mollusque busio.

Coquille facile à recomaitre à son ouverture orangée, et anx plis de son hord droil; recouverte de son drap marin, elle est d'un cendré blanchàre. De jeunes Balamus virent sourent sur celle espéce.

\section{Genre Nasse, Nissa Lam.}

27. Dasin eseanias Ibneg (Becciss).

Très-répandue dans tout l'archipel, mais surtout sur le littoral de San-Miguel et de Pico. - Varie beaucoup dans sa coloration.

28. Dassu geperula Broccu1. (Breciscm).

IIabite San-Miguel. Peu aboudante.

Se distingue de la précédenle par sa forme plus allongéc, moins ventrue, par ses côtes moins saillantes, et en général par un faciès plus élégant. Sa coloration est, le plus souvent, rosìtre ou roussàtre.

29. Dasnd Doeshayesll NoB. (Figurét).

Testa conico-acula, grosse costata, violacca cum fascris brumeis mimutis, crassu, solida; anfractibus $\mathrm{S}$; apcrturu ovali-acula, crossissima, candida, plicala; margine columellari reflexo, altero marginalo, 8-plicato.

Coquille conique-aiguë, munie de forles cùtes longitudinales, d'un gris-violacé, aree des linéoles brunes spirales faisant paraitre le lest conme strié-plissé, 
épaisse el très solide; huit tours de spire; ouverture ovale-aiguë, très-épaissie, blanche, plissée-crénelée, bord columellaire irès-réfléchi, hord droit muni d’un fort bourrelet et de huit plis. - IIauteur : 15, diamètre : 9 millimètres.

Mabite la côte méridionale de San-Higuel, sur les rochers submergés. Peu alondante.

Dédiée a M. Deshayes, continuateur des ourrages de Bruguière, de Férussac el de Lamarck, aulcur du Traite de Conchyliologie (ouvrage malheureusenent interrompu), l'un des premiers conchyliologistes de nolre époque.

$$
\begin{aligned}
& \text { Grure Caspere, Cassis Bruy. } \\
& \text { 30. Cussis sttleosin L.M. }
\end{aligned}
$$

Se rencontre de temps à antre ì Pico, sur la côte qui regarde Fayal. l'eu abondant.

\section{Genve Rocnen, Mrrex Lin.}

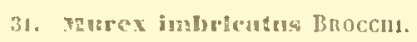

Coquille grisàtre, fortement plissée clans le sens de la spire, et munie en outre de grosses cótes longitudinales. L'ouverture est blanclie et le bord droit garni de plis nombreux. - Hauteur : 18, diamètre: 12 millimètres. - Elle paraît aussi voisine des liusus que des Murex.

Ilabite les côtes de Pico. Assez rare.

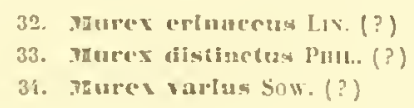

Ces trois espéces, recueillies avec ia précédente à 
Pico, dans le sable, n'ont pu ètre rigoureusement déterminées à cause de leur maurais élat de conservation. Ce n'est done qu'avee doute que je donne ces dénominations, surtout pour les deux dernières espèces.

$$
\begin{aligned}
& \text { Gente Tuton, Turtosion fabr. } \\
& \text { 35. Tritonium nodiferum leas. }
\end{aligned}
$$

La mer apporte quelquefois quelques beaux exemplaires de celle coquille sur les côtes de Pico, entre Area-Larga el Magdalena. Je l'ai recueillie aussi à San-Miguel, dans la baie de Rosto-de-Câo.

$$
\text { 36. Tritoniun serolbleubutor Liv. (Mtn:s). }
$$

Se trouve avec le précédent, à Pico.

$$
\begin{aligned}
& \text { Genre Colcminul., Colombelta Lam. } \\
& \text { 37. Coluubelln rustica Lix. (Voltth). } \\
& \text { Var. Amorlen Non. (Tigurie). }
\end{aligned}
$$

Cette variélé remarquable tient le milieu entre le type du Columbella rustica et le Columbella mercatoria. Fille est légèrement striće, marquée de nombrenses taches d'un brun-rougeâtre sur un fond jaunatre, quelquefois flammulée. Son ouverture est étroite et très-dentelée.

Très-commune dans tout l'archipel. Je l'ai recucillie abondamment sur les côles de Florès, de Graciosa, de San-Miguel, de Fayal et de Pico. Les femmes de Florès, qui sont très-coquelles, préparent avec ce coquillage différents ormements, notamment des collicrs. 
3. Colunbellu merentonla Lax. (Voteti).

Le fond de celte espece est blane avee des marbrures ou chinures, d'un brun-roux; elle est strice et méme sillonnće. Arec la précédente, mais beaucoup moins abondante.

\section{Genre Freac, Feses Lam.}

39.

Celle jolie coquille, laute de $S$ millimètres, large de quatre, est rouge de corail ou janne orangé, on mème linuàtre. Elle est trés-rugueuse, portant tout à la fois des stries transv ersales ed de fortes coites longitudinales. L'ourerture en est rose.

Elle labite les còtes de San-Miguel.

$$
\begin{gathered}
\text { Genre Mithe, Mithi Lam. } \\
\text { 40. Mitra lonecea Lau. }
\end{gathered}
$$

Très-abondante dans les parages de San-Miguel et de Pico.

Elle est ventrue dans toutes ses parties el aiguë au sommet. Son épiderme est d'un brun-olivitre. Sept tours de spire planes, a peine séparés par la suture, le dernier très-grand, formant plus de la moitie de la hauteur totale; cing plis sur la columelle. - Ilauteur : 35 , diamètre : 13 millimères.

\section{Genre Olive, Olwa ling.}

41. OHva ....?

Il a élé impossible de délerminer d'une manière 
précise l'espéce à lacuelle se rapporte un seul individu de ce genre, roulé ut décoloré, recucilli sur les cótes de Fayal. Sa taille el des vestiges de coloration le rapprochent des Olici candida et oriola.

\section{Gente Poncluaxi, Crumea lin. \\ 42. Cyprasu lumila Liv.}

Habite les côtes de Sauta-Maria, Fayal el Pico. N'est jas rare.

Celte espece est aisément reconnaissable à sa conleur gris-de-souris, à ses deux zônes transversales tres-pailes, et surtout aux deux taches noires qui se trouvent it chaque extrémití.

L.es femmes de Pico emploient ce coquillage en guise d'auneau à elefs. Elles le perforent à la partie antérieure, passent un fil el suspendent Jeur trousseau de clefs à cel anneau d'un nourcau genre.

$$
\text { 43. CรHrand ped]culus Lix. }
$$

Quelques rares exemplaires ont été rencontrés sur le littoral méridional de San-Mignel.

Coquille grisàtre, un peu rosée, avee quelques taches brunes; le sillon dorsal n'atteint pas les extrérnités.

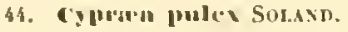

Habite, avec la précédente, a San-Nliguel; recueillie également sur le rivage occidental, à Pico.

Celte petite Porcelaine est d'un gris violacé en dessus, hanche en dessous; elle est élégamment striće sur les còtes, lisse à la partie supérieure. Longueur : 9 millimètres. 
45. Cypracen proalucta Gisli.

D'une taille encore plus réduite que la précédenle, cette Porcelaine est très-!」ombéc, élégamment striće dans toutes ses parties et enticrement blanche. Longueur : 8 millimètres.

Habite San-Niguel; recueillie sur la plage à Rostode-Cìo.

\section{Genre Haliotide, Hablotis Lin. \\ 46. Imbliotis tuberrulutu LiS.}

Très-abondante sur les côtes de Fayal et de Pico. On la trouve applicquéc contre les rochers submergés. Les Açoréens l'appellent lapa burra.

Animal noir; quand on l'irrite, il laisse échapper par loutes ses parties une humeur laiteuse. Suivant d'Orbigny (Mollusques des iles C'anaries, 1839, p. 95), on le mange aux Canaries et sur les cơtes de Bretagne: il n'est pas ćdule aux $A$ çores.

Le iest est presque toujours recouvert de nombreux parasites appartenant aux genres Serpula, l'ermelus, Vermilia, bialunus, et de polypiers microscopiques.

\section{7. aunllotis striula Las.}

Rencontrée sur les côtes de Fayal. Peu abondante.

Distincte de la précédente, en ce qu'elle n'olfre pas de tubercules entre les rides el les stries; n'est peut-être, cependant, 'qu'une variété. Elle est toujours moins grande. 
19. Hentudio cocelnen Ririt:

Ahoudante sur le littoral de San-Migucl. Recucillie également a rayal ef à lico.

Se distingue des précédentes par sa taille trèsinférieure, son test moins épais, un per rougreàtre, beaucoup moins rugueux. Ses plis spimax sont larges, fortement aceusés; ils sont laversés pát des stries tris-fines, surtout visibles dans la partic postćricure, vers le sommet. Sa nacre est fort billante et porte tres-risiblement l'empreinte des plis de la surface. - Longucur : 40-45, hauteur : $20-25$ millimètres.

\section{Genre Patemo, Patelua Lim.}

Observalion. - Toutes les espèces de ce genre sont édules aux Acores; elles sont connues vulgairement sous le nom de lapa. J'aurais pu en multiplier le nombre; mais j'ai préféré me tenir dans une prudente réserve et ne signaler que les types parfaitement distincts et nettement caractérisés.

\section{I'atella contel y'Onn.}

Coquille plus nu moins convexe suivant l'ige el la taille (plus convexe dans le jeune ìge), rugucuse, portant des rides longitudinales à peine apparentes, plus ou moins épaisse, mate, grisàtre, ou d'un cendré brunàtre; sommet asse\% aigu; péristôme formant nn ovale régulier; intérieur roussâtre, lisse, un peu nacré surtout vers le sommet. - Longueur : 60, dianıètre: 45 , hauteur : 20 millimètres. 
Habite les côtes de Santa-Mlaria, notamment la baie de San-Lourenzo. Peu abondante.

\section{6o. matella Ciomesii dion. (Figuren).}

Testa majuscula, subdepressa, rugosa, costulato-plicatula, solidula, extus grisco-brumea vel rufescens, intus nitide, brumea, margarilacea; vertice ad tertiam longihudinis partem sito, obtuso; aperhura ovali, integra.

Ilabite les rochers à fleur d'eau, à Santa-Maria (baie de San-Lourenzo) et à Pico. Rare.

Cette Patelle est assez grande, un peu déprimée, rugueuse, plisséc-còlcléc (plis et còtes plus ou moins apparents), peu épaisse; son sommet est oblus et rapproché du bord antérieur; l'épiderme (autant que l'on peut juger sous les végélations qui recourrent ces coquilles et qui varient leur coloration suivant leur nature) est d'un gris roussàtre ou brunâtre, plus rarement rougeàtre; l'ouverture est un ovale assez régulier, un peu arronli, quelquefois un peu atténué à la partie postéricure; l'intérieur est roussatre ou brunâtre, avec une couche légère de nacre bleuâtre, très-brillante. - Longucur : 50-60, diamètre : 50-53, hauteur : 12-15 millimètres.

Il est aisé de la distinguer de la précédente espèce à sa forme plus déprimée et moins allongée, à ses plis el à ses coutes plus saillants, enfin à l'état mousse et oblus de son sommet.

Je la dédie à M. Ie docteur Bernardino Antonio Gomès, premier médecin de Sa Majesté le Roi de Portugal, et botaniste distingué, comme un faible lémoiguage de mon sincère altachenent pour lui. 
Ilabile les côles de Pico. Rare.

Cetle Patelle est assez grande, plus ou moins épaisse, convexe, très-rugueuse, fortement plissée el côtelée; son péristòne est trés-sinneux el crénelé. Son épiderme est d'un brun-rougeàlre.

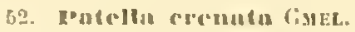

Abondante sur les côtes de Santa-Maria.

Coquille trés-élégaute, peu élerée, rayonnée de roussàlre el de brunitre, extrencment rugueuse, orméc de plis nombreux, imbrigućs, saillants, faisant parailre le test comme hérissé; son inléricur est bleware, sauf le fond qui est blanchatre; le púrislóme est crénclé el sinueux.

53. I"dellu movei v'Onb.

Commune a Pico el a Santa-Maria, sur les rochers du liltoral.

lille est plus grande que la précédente, roussâtre ou beunatre, presque loujours recouverte de fucus et de mousses marines, henucoup moins rugrueuse. Elle est fortement strice et plisséc, mais non lamelleuse ni hérisscic; l'inlćricur est bleuàtre avec unce grande tache blanche an fond, et quelquefois tout-afait blane. - Longucur : 76 , diamètre : 60 , hauteur : 20 millimètres.

Suivant le C'atalogue du docleur doln Jay ( $4^{e} e ́ d i-$ tion, 1 S52, 1. 100, 102798), le Palella . zorica Nutl. est icjentique à celle espece, qu'il indique comme se trouvant a Fayal. 
Testa majuscula, subelevalu, grosse costata, plicuta, crassa, solida, extus griseo-vividula, intus candidula; verlice subacuto, submedio; apertura oxuli, crenatula.

Corpuille assez grande, paksahlement élevée, trèsrugueuse, fortcment plisséc ế cûtelée, épaisse, solide; sommel conique, un peu aigu, pas trés-doigné du centre; épiderme grisitre on verdatte; ouverture ovalaire, avec les bords plissés et crénclés; nacre blanchàtre, arec une grande tache blanche rers te sommet. Cette latelle est sourent recourerte de balanes, de Serpules, de Vermets cl de disers fucus, de façon qu'il est assez difficile de se faire une idée exacte de sa coloration réelle. - Longucur : 60, diamètre: 50 , hauteur : 25 millimètres.

Habite les rochers à flemr d'eau, a Santa-Maria et à Pico. Assez ahoudante.

Je la dédie à mon excellent ami le docteur Baudon, de Mouy-de-l'Oise, auteur de différents travalux conelryliologiques, anatomiste et dessiualeur très-distingué. Cette dédicace n'est qu'un faible hommage rendu a l'amitić et au talcnt. Les planches qui accompagnent cet opuscule sont dues à son crayon labile et it son pinceau délicat.

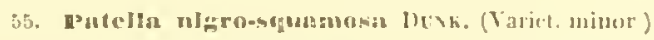

Dunlier rapporte que cette julie espèce a été découverle par le docteur Tams, aux envirous de Horta, ou elle est rare. Elle est d'm blanc sale ou jaunitre, et munic de petites côtes portant des ćeailles noires, comme hérissées. - Longncur : 2035 , diamètre : 15-20, hauteur : 8-12 uillimètres. 
(Conf. Dunker Index moll. Gmin. infer., 1853, p.41, $n^{0} 110$, tab.vi, fig. l, 5, 6, 7, 8, 9).

Je n'ai point rencontré celle cơnille, mais M. Mo. relet, mon compagnon de voyage, a rapporté une Patelle qui en est extrèmement voisine, simon idenlique.

50. Notella voveled Non. (Figure).

Testa subdepressa, rugosissima, costulato-imbricata, solidula, extus brumeo-viridula, intus subbrumea, margarilacea, nitidissima; verlice aculo; apertura ovala, plicata.

Habite les rochers des bords de la mer, a Fayal. Peu abondante.

Celle Patelle n'est pas grande, mais un peu surbaissée, très-rugueuse, fortement plissée-côtelée, avec les côtes cluargées d'aspérités imbriquées rendant le test àpre au toucher, comme une lime ou une râpe, peu épaisse ; l'épiderme est brunàtre, entrecoupé de tons verdàtres produits jar des régétations; l'ouverture est ovoïde, plissée-créncléc; nacre lorunâtre on roussâtre, avec un glacis irisé et une taclıe blanchàtre au sommet; sommel aigu, projeté en avant.

Lorgucur : 40, diamètre : 30 , latuteur : 12 millimètres.

Elle est voisine, mais cependant distincte, du Patella gramularis L. (teste el. Deshayes).

Découverte par M. Arthur Morelet, mon compagnon de voyage et ami, auteur de la Descriplion des Mollusques du Porlugal, du Voyage dans l'Amérique centrale, à qui je me fais un plaisir de la dédier. 


\section{Genre LotTis, Lotru Griy. \\ 57. Iottiu vilmined M LLL. (PATELAA).}

Coquille très-petite, blanchatre ou rosâtre, quelquefois blanche avec des rayons roses, ovale oblongue, assez élevée, avec le sommet dirigé en avant. Pour la forme, elle ressemble heanconp a un fort Ancylus. - Longuseur : 10, diamère : 7, hauteur : 4 millimètres.

IIabite Pico, sur le littoral occidental, entre Area. Larga el Magdalena.

\section{ACE'ERTERES.}

\section{Genre Hinite, IInisites Defi. \\ 58. Bnimutr.s MImusus GMet. (OSTREA).}

Habite en petit nombre les côtes de San Miguel et de Pico.

Espèce aisément reconnaissable aux difformités de ses valves. Rayons nombreux, serrés, rugueux. Couleur roussitre avec des taches violeltes.

$$
\begin{aligned}
& \text { Gemre Peigse, Pectex Brug. } \\
& \text { 59. Bocern pusio Lis. (Ostran). }
\end{aligned}
$$

Habite les côtes de San-Miguel. Commun.

Cette espéce est estrémemeul variable dans sa co- 
loration : elle est tantòt jaune, tantot orange, tantót rouge, souvent blanche, quelpuefois blanche et violetle, ou lirune, ou violacéc, souvent mouchetée de violet, de rouge ou de brun, avec des passages entre toutes ces nuances. Elle n'atteinl pas une forte taille, el sa forme génćrale est assez constante.

60. mecten molulifer Sow.

Habite San-Mignel. Peu abondant.

Jolie espece, le plus souvent rouge ou rougeàtre, striée-plissée, portanl de grosses côtes inégales, sinueuses el comme noueuses.

Geme Lame, Lima Brug.

61. Ihua tencra Tent.

Coquille blanche, allongéc, un peu translucide, fragile, finement et élégramment rayonnée, à bords crénelés. - Longueur : 25, hauleur : 14 millimètres.

Habite San-Miguel.

\section{Geure Pehne, Penxa Brug.}

62. กอบ ....

Rang (Manuel, 1829, J. 253) indique, sans la nommer, une espece de ce genre comme se trouvant aux Açores el aux îles du Cap-Vert. Je n'ai pas été assez heureux pour la rencontrer.

\section{Geure Aricule, Avicula Brug. 63. Micula Tarentlun LaM.}

Jolie espèce, à forme oblique et à test mince, fra- 
gile, grisàtre, transparente avec des rayons Jruns; aile obliquement arrondie; valves de grandeur égale, couvertes d'un grand nombre de petites pointes écailleuses. M. Morelet l'a vue sur des polyjpiers péchés sur les côtes de Fayal.

\section{Avipula Mtantien LuY.}

Coquille brunàtre, à valves inégales; aile large, arrondie, à peine oblique.

Avec la précédente et dans les mèmes conditions.

Genre Arcue, Arra Lin.

65. Areu nasieularis Brug.

IIabite les côtes de Pico el de San-Miguel. Assez rare. - L'Arca tetragona, de Lamarck, parait identique à celte espèce.

\section{Gente Pixice, Pixia Lin.}

66. pinna rudis Lix.

Quelques individus ont été recueillis sur les còles de San-Miguel el de Pico. C'est la plus grande coquille marine de ces parages.

Lors de mon passage à Lisbonne, au retour de mon exploration des Açores, j’ai eu l'lıonneul de remettre un magnifique exemplaire de cette espèce à S. M. le Roi de Portugal, qui a bien voulu l'accepter pour son Musée particulier. Depuis mon retour en France, j'ai également eu l'honneur d'arlresser à Sa Majesté, de concert avec M. Morelet, une collection des coquilles tant terrestres que marines de l'archipel des Açores, collection destinée au Muscee du Roi. 


\section{Geme Canute, Camuta Brug. \\ 67. Corritu simunta Bacg.}

Ilabile Pico, sur la plage, entre Area-Larga et Magdalena. Rare. Plus aboudante a San-Mignel, sur la coile du sud.

Cotjuille grisitre on blanchatre, avec des bandes brunes transversales, sinucuse inférienrement; coles très-élerées, rugueuses, imbriquées; intéricur blane et lorun.

\section{Gemic Bucanne, Cannum Lin. \\ Gs. Covilum funciutum Movt.}

Habite les eóles de San-Miguel. Très-commune.

Putite coquille globuleuse, subarrondic, tris-solide, blanche avec des zònes roussotres, munie de eôtes larges, aplaties, portant de petits tubercules en avant et en arriere, et dont les interstices sont ponctués; crochets aigus, recourbés; intérieur blanc et violet, strici sur les bords.

$$
\begin{aligned}
& \text { Gente Envise, Envisa Turt. } \\
& \text { 69. Erollin enstunen Mont. (1)oxax). }
\end{aligned}
$$

'Très-commune sur la côte méridionale de SanMiguel.

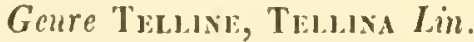

$$
\begin{aligned}
& \text { 70. Tullnu incurnutu Lix. }
\end{aligned}
$$

Très-abondante à Sau-Miguel, sur le lilloral méridional.

Jolic espece trés-aplatie, lisse, luisante, blanche 
en delıors, le plus souvent orangée ou sammonée en dedans (quelquefois toute blanclie ou toute saumonée), trés-faiblement rayonnée. Sa longueur est de 30 à 35 millimètres.

\section{Genre Crthénée, Crthenei Lam. \\ 71. Cothenen Chtone Lis. (VEnes).}

De jeunes exemplaires ont été récoltés â San-Mligucl, arec les espèces qui précúdent. Peu abondanic.

\section{Genre Solex, Solex Lin. 72. Solen manginatus Pelt.}

Ilabite les côtes de San-Miguel; assez rare. Longueur : 10 centimétres.

\section{CIIR IRT OPOHES.}

Gente Avatife, Anatea Brug.

73. Anutifa lurvis LaM.

IJabile Graciosa el San-Miguel; ahondante.

Pendant une traversée entre les îles de Graciosa el de Florès, dans le courant de juillei, j’ai été à mème de constater avec quelle abondance celte espèce se multiplie au milieu de certaines circonstances favorables. Les matelots du yacht sur lequel j'étais 
monté (le Santa-Cruz, de Fiyal) aperçurent une énorme poutre flottante, amenée sins donte des côtes d'Amérique pal le Gulstream (ou débris de quelque navire naufragé), et s'empressèrent le la capturer. Quand cette poutre fut lissée sur le pont, quelle ne fut pas la surprise de tout l'équipage en recomaissant qu'elle était recouverte de toutes parts par des masses compactes d'Anatifes! Ces anmaux étaient tellement serrés les uns contre les autres, qu'il eût été impossible d'en placer cle nouveaux individus, à moins de les superposer, et cela, sur toutes les faces du madrier. J'en mis un bou nombre dans l'esprit-de-vin, aprés quoi les matelots s'élant armés de hachettes el de comeanx, débarrassèrent leur prise de sa bizarre enveloppe. Ils appellent l'A. natife buzano. Sur ce singulier asile vivaient également des ammélides, de petits crabes, et d'autres animaux inférieurs.

\section{Geme Balave, Balaxus Brug.

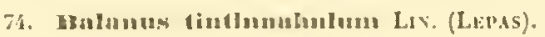

Trés-abondant sur les rochers des còtes de tout l'Archipel, mais surtout à San-lliguel.

Les Açoréens sont très-friands de ce mollusque, qu'ils appellent vulgairement craca; ils le mangent après l'avoir fait cuire simplenent a l'ean avec un peu de sel. J'ai goûté de ce mets par curiosité; mais je déclare que, bien que je lui aie trouré un peu de ressemblance pour le goùt avec les crevettes, je n'ai pu partager leur engoument pour lui. Cependant les amateurs prétendent que c'est un mets fort agréable, et c'est comme tel qu'il nous fut offert clans plusicurs 
localités. A Iiurnas (île de San-llignel), on en fail une grande consommation à l'épocque de la saisou des bains : on les tire de Ribeira-Quente, qui a la réputation de les produire meilleurs que sur les autres points de l'ile.

Dans les cavilés qui se lrourent autour des groupes de Balanes, j’aj souvent remarqué de jeunes in. dividus de l'Échinus brevispinosus Risso.

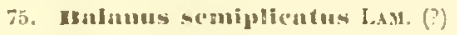

Très-petile espèce, courle, ramassée, còteléc, vivant par groupes nombreux sur les rochers voleaniques de l'ilot de Villa-Franca (San-Mignel). Je ne suis pas tout-à-fait certain de cette détermination spécificue. 



\section{APPENIICE.}

\section{7lollasuges fosgiles.}

Sur les neuf ìles de l'archipel des $\Lambda$ çores, huit semblent être exclusivement le résultat d'une action volcanique sous-marine. Celles-ci portent à leur surface l'empreinte irrécusable de leur origine, et che\% plusieurs encore, l'action des feux souterrains se manifeste au dehors par des phénoménes fort remarquables. C'est ainsi que le fameux Pic de l'ile de Pico, cône immense qui ne mesure pas moins de 7,000 pieds de lıat, fume encore quelquefois et dégage presque constamment des vapeurs sulfuriques et des gaz inflammables. C'est ainsi encore que le voyageur admire, non sans émotion, dans le val de Furnas, à San-Miguel, des volcans d'eau bouillante en pleine activité, dont les vapeurs brùlantes entretiennent dans la vallée une liumidité permanente, et qui s'échappent quelquefois de leurs gouffres souterrains avec un bruit terrible, reconvant le sol brùlant et presque mouvant, qui les environne, de dépôts de soufre el de stalagmite siliceuse.

Les éléments géologiques composant les chaines de montagnes de ces îles et leurs masses énormes de rochers sont : le basalte, le trachyte, des laves, des pierres ponces, des conglomérats de scories, de 
ponce et d'olssidienne, des tufs variés. I.es roches et les lares basaltiques ef lrachyliques comtiement toutes plus ou moins de pyroxène, d'amphibolie, d'olivine el de mica; mais aucune trace, lien entendu, de corps organisćs fossiles.

La gracieuse ile de Saxta-Mana, la plus méridionale de toutes $\left(36^{\circ} 50^{\prime}\right.$ de latilude $\mathbf{N}$.) el la plus rapprochée du continent, présente seule un autre aspect el des conditions géologiques qui sembleraient indiquer, ou une origine différente, ou la combinaison chez elle de certains phénomènes de natures diverses, qui auraient sensiblement modifié sá structure el sa composition. Ce qui porta certains géologues à la considérer comme une dépendance de l'île Madère, tandis que d'autres la regardent simplement comme un prolongement de la partie orientale de San-Miguel. Quoiqu'il en soit, la hase des roches est encore le basalte ot le trachyte : mais elle ne porte point à sa surface les traecs récentes de l'effort des feux souterrains, ni courant de lave, ni cratère de volean. De plus, j'y ai rencontré, en plusieurs localités el sur un grand espace, des couclies assez puissantes de terrain calcaire, abondamment pourvues de nombreux fossiles.

Malbeureusement ces roches calcaires sont extrèmement dures el compactes. Dépourvu des instruments nécessaires, et donnant d'ailleurs tous mes soins à la zoologrie et à la bolanique, je n'ai pu recueillir qu'un très-petit nombre de fossiles, lit plupart en mauvajs étal de conservation el presque mécomnaissables. Voici les seules espèces que j'aie pu reconmaître d'une façon certaine, et dont je juis garantir la délermination comme exacte : à l'exception 
toutefois des Anomia ephippium et Cardium lovigatum, qui me laissent encore dans le doute. J'ai soumis le tout à l'examen de M. Deslayes, et aussi à celui de M. Bronn, professeur à Heidelberg.

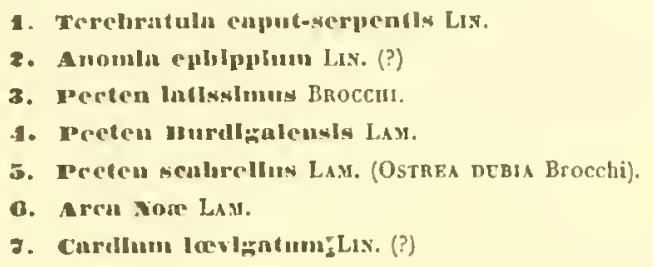

La présence du Pecten latissimus, propre jusqu'ici au terrain tertiaire supérieur de l'Italie et des autres points du littoral méditerranéen, n'indiquerait-elle pas une corrélation d'àge entre eertaines couches du terrain de Santa-Maria et le terrain subapennin?... On pourrait être tenté de former cette hypothèse. Cependant, me dit M. Deshayes (à propos de mes fossiles), en considérant ces formes dans leur ensemble, on reconnaîl aisément qu'elles appartiennent à des espèces récentes, et que le terrain où elles gisent doit êlre très-moderne.

Tels sont les seuls documents que je puisse à présent fournir sur les mollusques marins fossiles de Santa-Maria. L'un de mes compagnons de voyage, M. Hartung, s'occupant exelusivement de géologie, a recueilli des échantillons plus nombreux et aussi en meilleur état de conservation. Ces spécimens ont été remis à II. Bronn, d'Heidelberg, qui a reconnu bon nombre d'espéces inédites, et qui se propose, si je suis bien informé, de publier prochainement un mémoire spécial sur cet intéressant sujet. 

EXPLICATION DES PLATCHES. 


\section{PLANCIE PREMIJ̈E.}

Fig. 1-2, Litiopa Grutelupeana Drouët.

Fig. 3-4, Nnssa Deshayesii Drouët.

Fig. 5, Columbella rustica, var. Azorica.

Fig. 6-7, Patella Gomesii Drouët. 


$$
0^{\circ}
$$



PLANCHE DEUXILYE.

Fig. 8-9, Patella Baudonii Drouët.

Fig. 10-11, Patella Moreleti Drouët. 


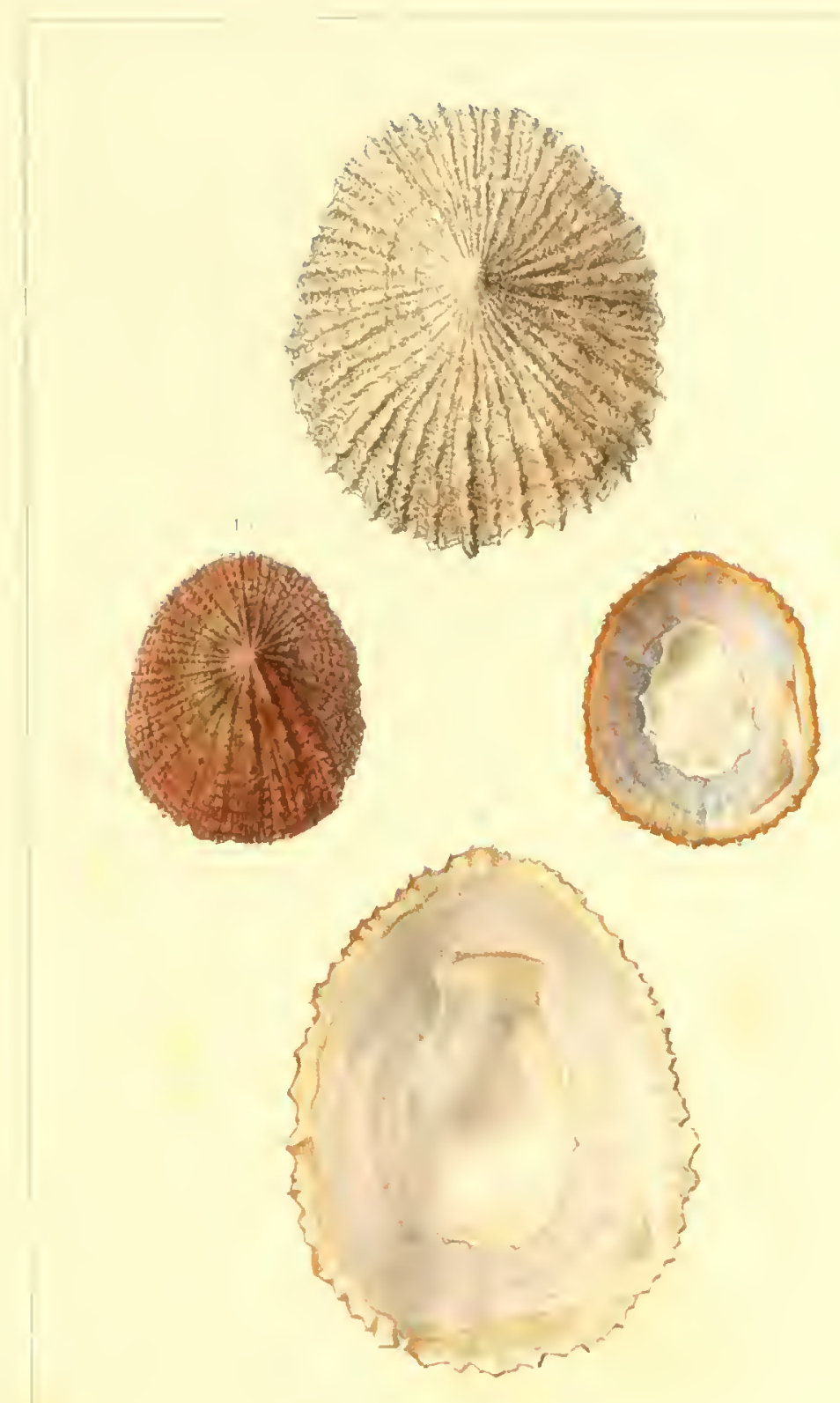



Extrait des falémuires de la Société Acadrmique de l'Aube. Tome XXII, 1858. 








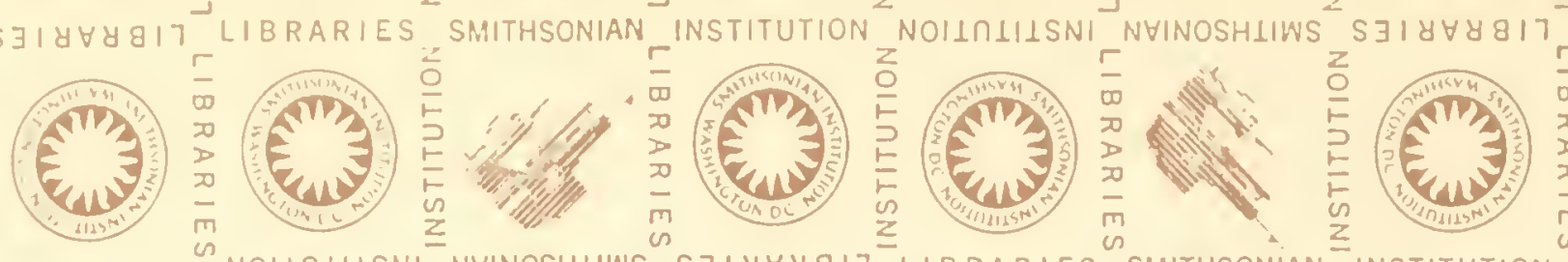
INSTITUTION NOILOIILSNI NVINOSHIIWS S I I $\forall Y 817$ LIBRARIES SMITHSONIAN INSTITUTION
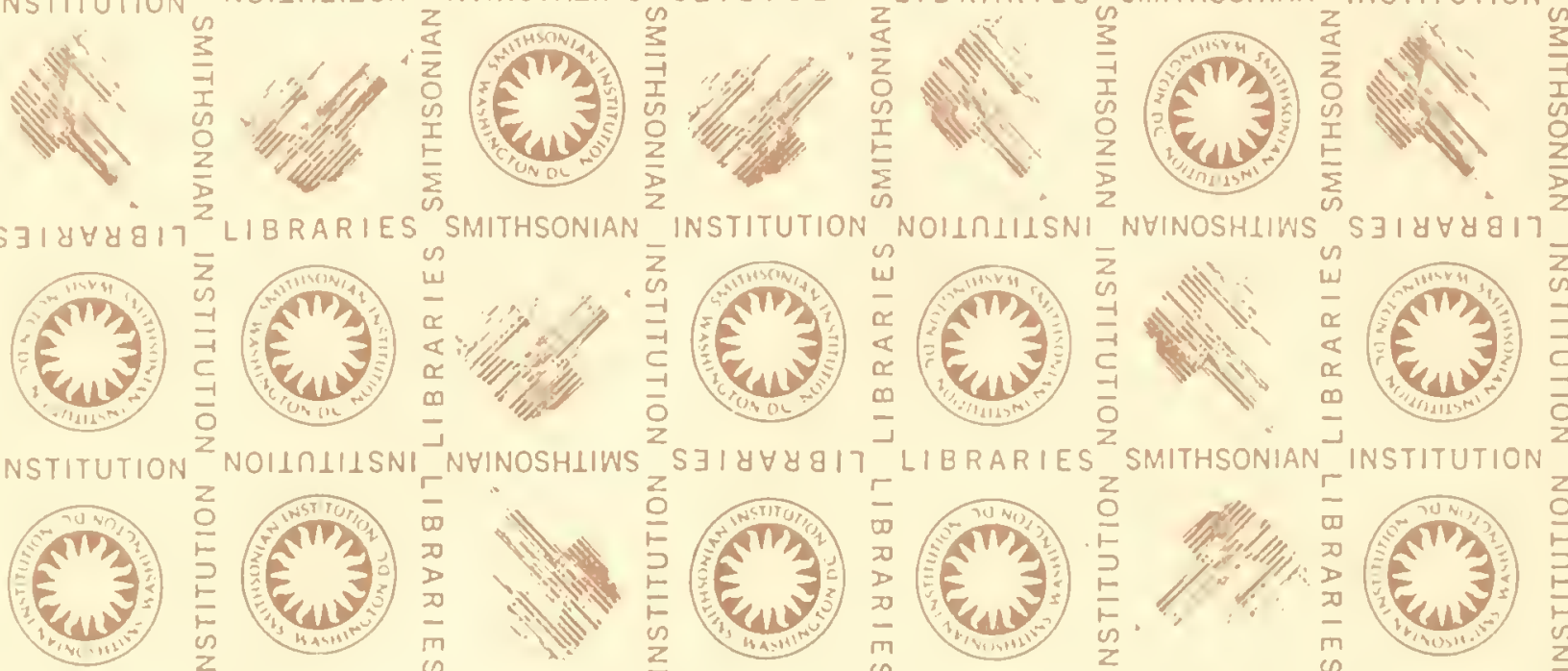
ELRRARIES SMITHSONIAN INSTITUTION NOHOHHSNI
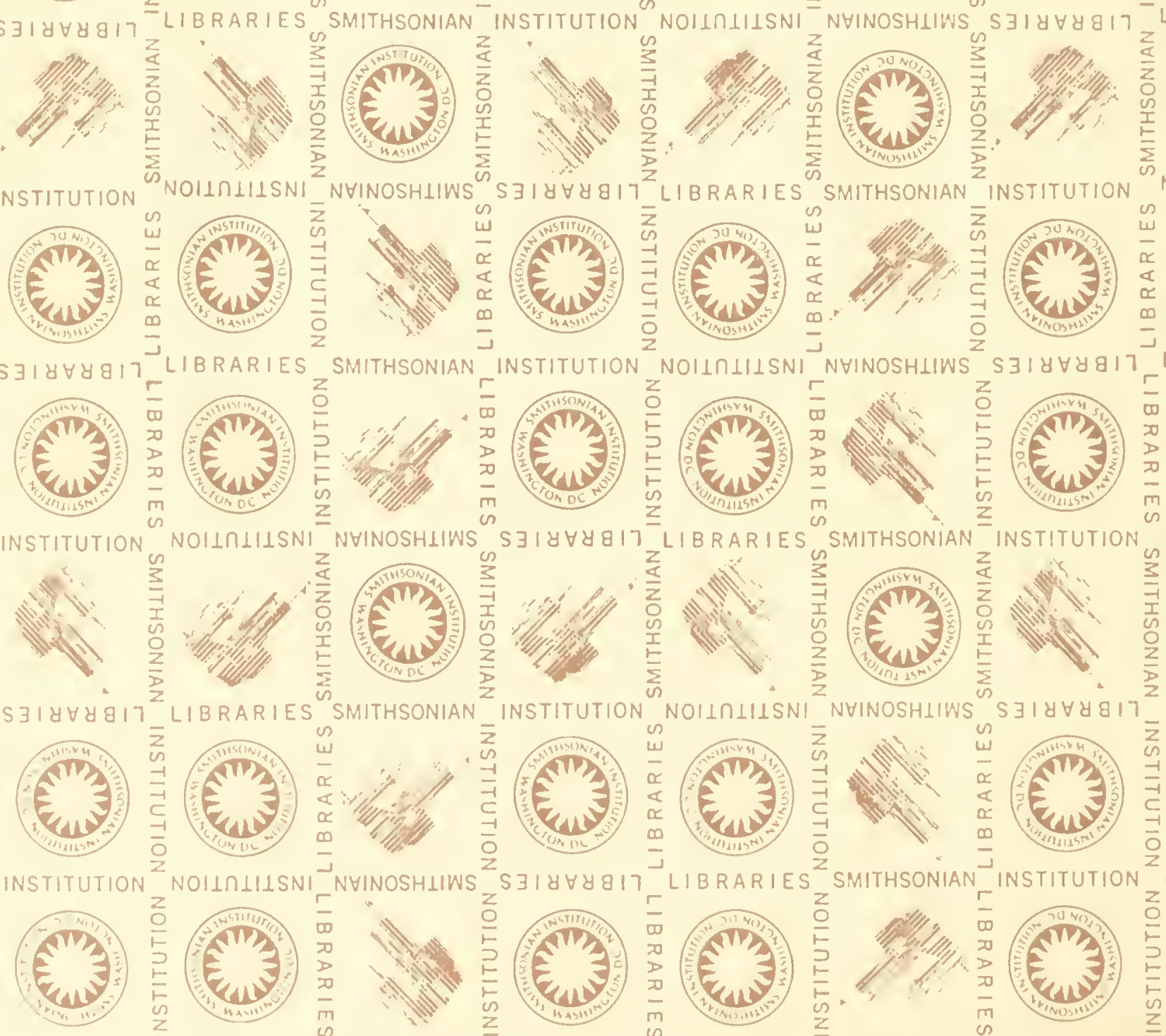


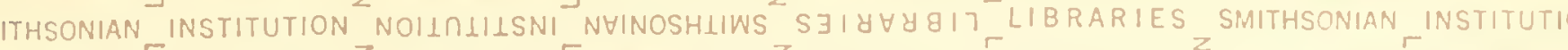
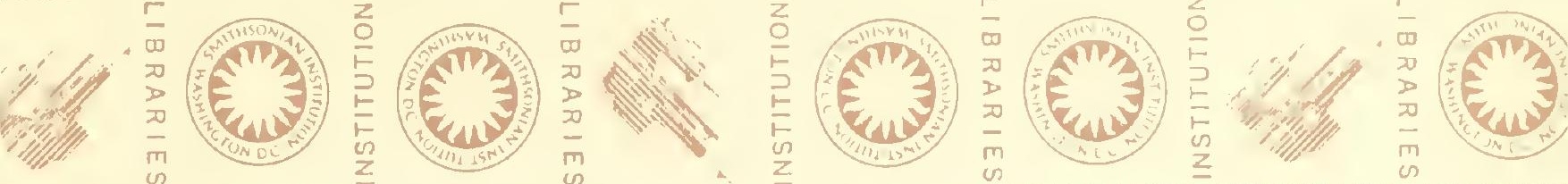
(n)
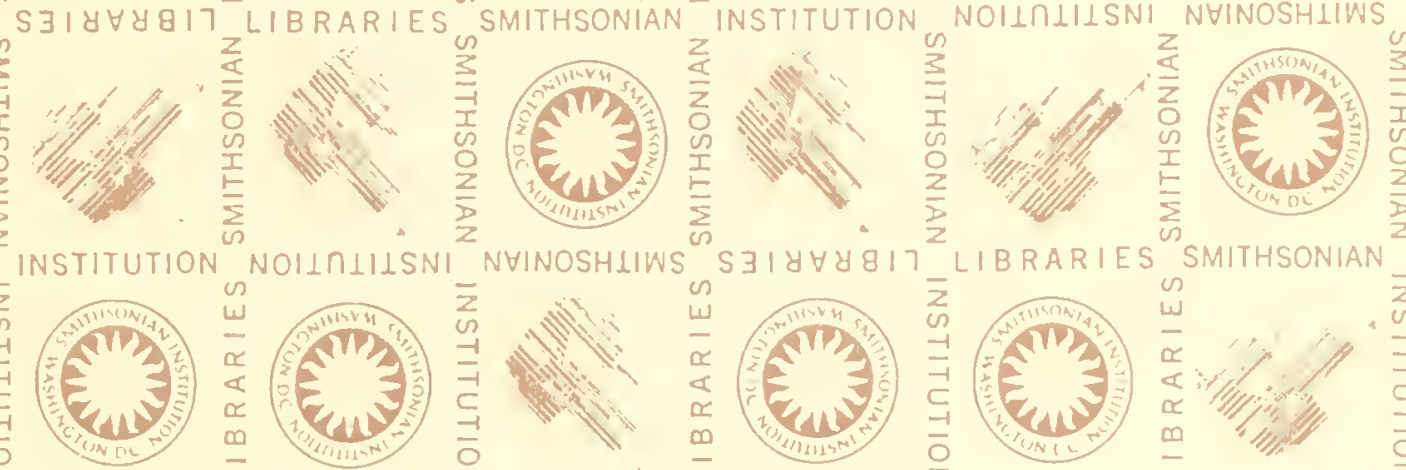

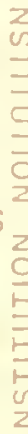
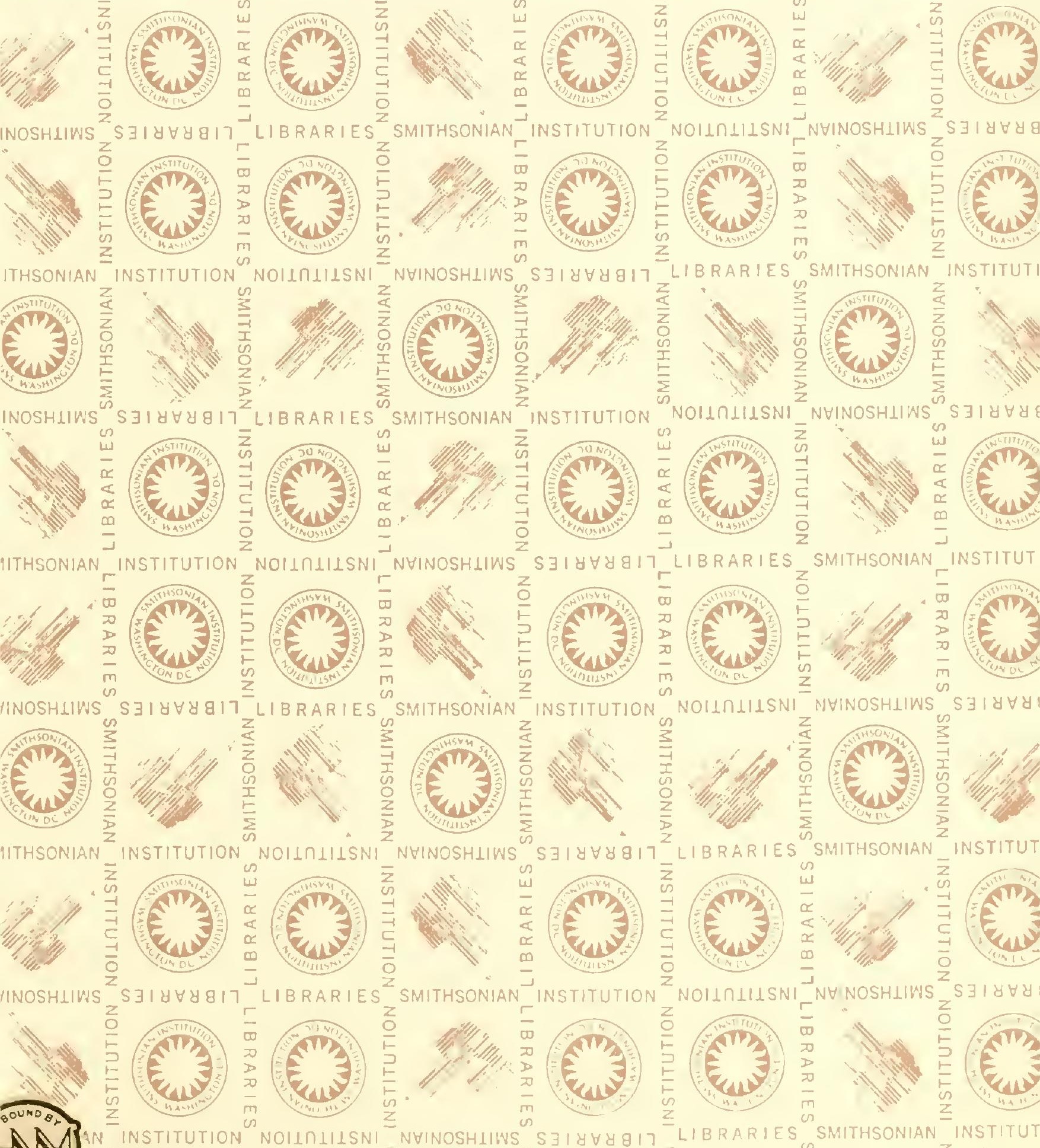
